\title{
An Adaptive Algorithm for Ordinary, Stochastic and Partial Differential Equations
}

\author{
Kyoung-Sook Moon, Erik von Schwerin, Anders Szepessy, and Raúl \\ Tempone
}

\begin{abstract}
The theory of a posteriori error estimates suitable for adaptive refinement is well established. This work focuses on the fundamental, but less studied, issue of convergence rates of adaptive algorithms. In particular, this work describes a simple and general adaptive algorithm applied to ordinary, stochastic and partial differential equations with proven convergence rates. The presentation has three parts: The error approximations used to build error indicators for the adaptive algorithm are based on error expansions with computable leading order terms. It is explained how to measure optimal convergence rates for approximation of functionals of the solution, and why convergence of the error density is always useful and subtle in the case of stochastic and partial differential equations. The adaptive algorithm, performing successive mesh refinements, either reduces the maximal error indicator by a factor or stops with the error asymptotically bounded by the prescribed accuracy requirement. Furthermore, the algorithm stops using the optimal number of degrees of freedom, up to a problem independent factor.
\end{abstract}

\section{Introduction to the Adaptive Algorithm}

This work presents an overview of the authors work on the convergence rate of an adaptive algorithm to compute functionals of solutions to ordinary, stochastic and partial differential equations. The main ingredient of the adaptive algorithm is an error expansion of the form

$$
\text { Global error }=\sum \text { local error } \cdot \text { weight }+ \text { higher order error, }
$$

with computable leading order terms. The weight is the sensitivity of the functional of the solution with respect to perturbations in the differential equation. For an ordinary differential equation, the error expansion (11) can be derived by the variational principle in $[\mathbf{9}]$ and for weak approximation of stochastic differential equations the error expansion (11) can be derived based on computable stochastic

1991 Mathematics Subject Classification. 65L50,65C30, 65N50.

Key words and phrases. adaptive methods, mesh refinement algorithm, a posteriori error estimate, computational complexity.

Support from the Swedish Research Council grants 2002-6285 and 2002-4961, UdelaR in Uruguay, a Swedish Foundation for Strategic Research grant and the European network HYKE, funded by the EC as contract HPRN-CT-2002-00282, is acknowledged. 
flows and discrete dual backward problems in [12]. For partial differential equations, [6] derives an asymptotic expansion of the error using the dual weighted residual method.

The goal of the adaptive algorithm for differential equations based on these error expansions, is to approximate the desired quantity of interest with an adapted mesh using as few mesh elements as possible, for a given level of accuracy using a given approximation method, for instance the Euler method or piecewise bilinear finite elements with varying mesh size. Based on the a posteriori error expansions of the form (11) the global error can be asymptotically approximated by

$$
\text { Global error } \approx \sum_{\mathcal{K}} \text { error indicator, }
$$

where $\mathcal{K}$ is a set of time steps or elements. A typical adaptive algorithm does two things iteratively :

(i) if the error indicators satisfy an accuracy condition, then it stops; otherwise

(ii) the algorithm chooses where to refine the mesh, recomputes the error indicators and then makes an iterative step to (i).

Therefore the indicators not only estimate the localization of the global error but also give information on how to refine the mesh in order to achieve optimal efficiency.

Despite the wide use of adaptive algorithms and the well developed theory of a posteriori error estimates, less is known theoretically on the behavior of adaptive mesh refinement algorithms, see $[\mathbf{8}, \mathbf{6}, \mathbf{1 0}]$ for brief overviews of previous work. To introduce the main ingredients, let us consider a simple integration problem, namely for a given function $X:[0, T] \rightarrow \mathbb{R}$ approximate the integral $g(X)=\int_{0}^{T} X(t) d t$. Let us first discretize the time interval $[0, T]$ into $N$ subintervals $0=t_{0}<t_{1}<\cdots<$ $t_{N}=T$ with corresponding time steps $h_{n}:=t_{n+1}-t_{n}$. Now, if we approximate $g(X)$ using the left point rule (forward Euler) denoted by $\bar{g}$, our global discretization error becomes

$$
\text { Global Error }=g(X)-\bar{g}=\sum_{n=0}^{N-1}\left(h_{n}\right)^{2} \rho_{n}+\text { higher order terms, }
$$

with the error density function $\rho$ defined by $\rho_{n}:=\frac{d X}{d t}\left(t_{n}\right) / 2$. From the definition of the number of time steps

$$
N(h):=\int_{0}^{T} \frac{1}{h(\tau)} d \tau,
$$

the number $N_{u}$ of uniform steps to reach a given level of accuracy TOL turns out to be asymptotically proportional to the $L^{1}$-norm of the function $\rho$, i.e.

$$
N_{u} \simeq \frac{T}{\mathrm{TOL}}\|\rho\|_{L^{1}(0, T)} .
$$

On the other hand, by minimizing the number of steps in (ब1 subject to an accuracy constraint, i.e. imposing the leading order of (3) to be TOL, a standard Lagrangian relaxation yields the optimal choice

$$
h_{n}^{2} \rho_{n}=\text { constant for all } n
$$


and as a consequence the number $N_{a}$ of adaptive steps becomes proportional to the smaller $L^{\frac{1}{2}}$ quasi-norm of the error density, i.e.

$$
N_{a} \simeq \frac{1}{\mathrm{TOL}}\|\rho\|_{L^{\frac{1}{2}}(0, T)} .
$$

For instance, if we have a singular case, $X(t)=1 / \sqrt{t}$, we can instead compute with $X_{\epsilon}(t)=1 / \sqrt{t+\epsilon}$, choosing the positive parameter $\epsilon$ such that

$$
\left|\int_{0}^{T}\left(X(t)-X_{\epsilon}(t)\right) d t\right|=o(\mathrm{TOL})
$$

i.e. $\epsilon^{1 / 2} \lesssim o$ (TOL). Therefore, the number of uniform time steps becomes

$$
N_{u} \simeq \frac{T / 4}{\mathrm{TOL}} \int_{0}^{T} \frac{d t}{(t+\epsilon)^{3 / 2}} \simeq \frac{T / 4}{\mathrm{TOL}} \frac{1}{\epsilon^{1 / 2}} \gtrsim \mathcal{O}\left(\mathrm{TOL}^{-2}\right)
$$

while the number of adaptive time steps is the smaller

$$
N_{a} \simeq \frac{1 / 4}{\mathrm{TOL}}\left(\int_{0}^{T} \frac{d t}{(t+\epsilon)^{3 / 4}}\right)^{2} \approx \mathcal{O}\left(\mathrm{TOL}^{-1}\right)
$$

which clearly shows the advantage of an adaptive approach. In the sequel we will consider multiscale problems, i.e. problems that have very different scales so that the error density, although being bounded, may be very large, e.g. $\frac{1}{\epsilon^{3 / 2}}$.

Thus, having motivated the need for adaptivity in the previous example, we now state the main questions to answer, namely

What is the notion of error density for ordinary, stochastic and partial differential equations?

What is a suitable approximation for such an error density?

What can be concluded about the convergence rate of the adaptive algorithm?

In this paper, Section 2 describes an adaptive algorithm based on previously

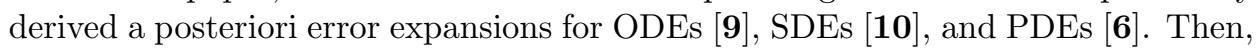
Section 3 presents results on the convergence rates of the adaptive algorithm.

\section{Convergence of the Error Density and the Adaptive Algorithm}

2.1. An Error Expansion for ODEs. Let us consider an ordinary differential equation (ODE) of the form

$$
\frac{d X(t)}{d t}=a(t, X(t)), 0<t \leq T,
$$

with an initial value $X(0)=X_{0} \in \mathbb{R}^{d}$ and a given flux $a:[0, T] \times \mathbb{R}^{d} \rightarrow \mathbb{R}^{d}$. First discretize the time interval $[0, T]$ into $N$ subintervals $0=t_{0}<t_{1}<\ldots<t_{N}=T$ and let $\bar{X}$ be an approximation of $X$ in (5) by any $p$-th order accurate numerical method, satisfying the same initial condition $\bar{X}(0)=X(0)=X_{0}$.

We are interested in computing a function value $g(X(T))$ for a given general function $g: \mathbb{R}^{d} \rightarrow \mathbb{R}$, which may represent the quantities of physical interest. Using the variational principle, we show in $[\mathbf{9}]$ that the global error has the expansion

$$
g(X(T))-g(\bar{X}(T))=\sum_{n=1}^{N}\left(\bar{e}\left(t_{n}\right), \bar{\Psi}\left(t_{n}\right)\right)+\int_{0}^{T} o\left(h^{p}(t)\right) d t
$$


where $(\cdot, \cdot)$ is the standard scalar product on $\mathbb{R}^{d}$. Here the approximate local error is defined by $\bar{e}\left(t_{n}\right):=\gamma\left(\bar{X}\left(t_{n}\right)-\bar{X}\left(t_{n}\right)\right)$, where $\gamma$ is a Richardson extrapolation constant and the approximate local exact solution $\bar{X}$ is computed with smaller time steps or a higher order method than $\bar{X}$. The weight $\bar{\Psi}$ is an approximation of $\Psi$, which solves the dual equation

$$
\begin{aligned}
-\frac{d \Psi(s)}{d s} & =\left(a^{\prime}\right)^{*}(s, X(s)) \Psi(s), s<T \\
\Psi(T) & =g^{\prime}(X(T))
\end{aligned}
$$

where $\left(a^{\prime}\right)^{*}(s, x)$ is the transpose of the Jacobian matrix.

Therefore the leading order term in (6) has the approximate error density

$$
\bar{\rho}_{n}:=\frac{\left(\bar{e}\left(t_{n}\right), \bar{\Psi}\left(t_{n}\right)\right)}{h_{n}^{p+1}},
$$

which is then used in the adaptive algorithm, see Section 2.4.

2.2. An Error Expansion for SDEs. Let us consider an Itô Stochastic differential equation (SDE) of the form

$$
d X_{k}(t)=a_{k}(t, X(t)) d t+\sum_{\ell=1}^{\ell_{0}} b_{k}^{\ell}(t, X(t)) d W^{\ell}(t), t>0,
$$

where $k=1, \ldots, d$ and $(X(t ; \omega))$ is a stochastic process in $\mathbb{R}^{d}$, with randomness generated by the independent one dimensional Wiener processes $W^{\ell}(t ; \omega), \ell=1, \ldots, \ell_{0}$, on the probability space $(\Omega, \mathcal{F}, P)$. The functions $a(t, x) \in \mathbb{R}^{d}$ and $b^{\ell}(t, x) \in \mathbb{R}^{d}$, $\ell=1, \ldots, \ell_{0}$, are given drift and diffusion fluxes.

The goal is to construct approximations to the expected value $E[g(X(T))]$ by a Monte Carlo method, for a given function $g: \mathbb{R}^{d} \rightarrow \mathbb{R}$. Examples of such an expected value are the computation of option prices in mathematical finance, stochastic climate prediction, wave propagation in random media, etc. The Monte Carlo Euler method approximates the unknown process $X$ by the Euler method $\bar{X}\left(t_{n}\right)$ which is a time discretization based on the nodes $0=t_{0}<t_{1}<\cdots<t_{N}=T$ where

$$
\bar{X}\left(t_{n+1}\right)-\bar{X}\left(t_{n}\right)=h_{n} a\left(t_{n}, \bar{X}\left(t_{n}\right)\right)+\sum_{\ell=1}^{\ell_{0}} \Delta W_{n}^{\ell} b^{\ell}\left(t_{n}, \bar{X}\left(t_{n}\right)\right),
$$

and $h_{n} \equiv t_{n+1}-t_{n}, \Delta W_{n}^{\ell} \equiv W^{\ell}\left(t_{n+1}\right)-W^{\ell}\left(t_{n}\right), n=0,1,2, \ldots, N-1$. The aim of the adaptive algorithm is to choose the size of the time steps, $h_{n}$, and the number of independent identically distributed samples $\bar{X}\left(\cdot, \omega_{j}\right), j=1,2, \ldots, M$, such that the computational work, $N \cdot M$, is minimal while the approximation error is bounded by a given error tolerance, TOL, i.e. the event

$$
\left|E[g(X(T))]-\frac{1}{M} \sum_{j=1}^{M} g\left(\bar{X}\left(T ; \omega_{j}\right)\right)\right| \leq \mathrm{TOL}
$$

has a probability close to one. A priori error estimates of the computational error

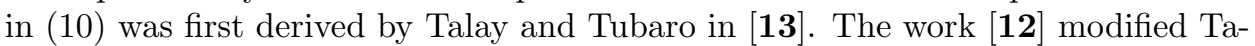
lay's and Tubaro's error expansion to an expansion with computable leading order term in a posteriori form, based on computable stochastic flows and discrete dual 
backward problems. Stopped diffusion, including for example the barrier option, is an example where adaptive time steps improve the convergence rate, see [ $\mathbf{7}]$.

Assume that the process $X$ satisfies (8) and its approximation, $\bar{X}$, is given by (9), we have, see [12, 10]

TheOREM 2.1 (Error expansion for SDEs). Suppose there are positive constants $k$ and $C$ and an integer $m_{0}$ with the bounds

$$
\begin{aligned}
& g \in \mathcal{C}_{\text {loc }}^{m_{0}}\left(\mathbb{R}^{d}\right),\left|\partial_{\alpha} g(x)\right| \leq C\left(1+|x|^{k}\right), \text { for all }|\alpha| \leq m_{0}, \\
& E\left[|X(0)|^{2 k+d+1}+|\bar{X}(0)|^{2 k+d+1}\right] \leq C,
\end{aligned}
$$

and

$$
a \text { and } b \text { are bounded in } \mathcal{C}^{m_{0}}\left([0, T] \times \mathbb{R}^{d}\right) \text {. }
$$

Assume that $\bar{X}$ is constructed by the forward Euler method with step sizes $h_{n}$ produced by the stochastic time step version of the adaptive algorithm in Section 2.4 and the corresponding $\Delta W_{n} \equiv W\left(t_{n+1}\right)-W\left(t_{n}\right)$ are generated by Brownian bridges. Assume also that $\bar{X}(0)=X(0)$ and $E\left[|X(0)|^{k_{0}}\right] \leq C$ for some $k_{0} \geq 16$. Then the time discretization error has the expansion

$$
\begin{aligned}
E[g(X(T))- & g(\bar{X}(T))]=E\left[\sum_{n=1}^{N} \bar{\rho}_{n}\left(h_{n}\right)^{2}\right] \\
& +\mathcal{O}\left(\sqrt{\frac{\mathrm{TOL}}{c(\mathrm{TOL})}}\left(\frac{C(\mathrm{TOL})}{c(\mathrm{TOL})}\right)^{8 / k_{0}}\right) E\left[\sum_{n=1}^{N}\left(h_{n}\right)^{2}\right]
\end{aligned}
$$

with computable leading order terms, where

$$
\begin{aligned}
\bar{\rho}_{n}\left(t_{n}, \bar{X}\right) \equiv & \frac{1}{2}\left(\frac{\partial}{\partial t} a_{k}+\partial_{j} a_{k} a_{j}+\partial_{i j} a_{k} d_{i j}\right) \varphi_{k}\left(t_{n+1}\right) \\
& +\frac{1}{2}\left(\frac{\partial}{\partial t} d_{k m}+\partial_{j} d_{k m} a_{j}+\partial_{i j} d_{k m} d_{i j}+2 \partial_{j} a_{k} d_{j m}\right) \varphi_{k m}^{\prime}\left(t_{n+1}\right) \\
& +\partial_{j} d_{k m} d_{j r} \varphi_{k m r}^{\prime \prime}\left(t_{n+1}\right),
\end{aligned}
$$

and the terms in the sum of (12) are evaluated at the a posteriori known points $\left(t_{n}, \bar{X}\left(t_{n}\right)\right)$, i.e.

$$
\partial_{\alpha} a \equiv \partial_{\alpha} a\left(t_{n}, \bar{X}\left(t_{n}\right)\right), \partial_{\alpha} b \equiv \partial_{\alpha} b\left(t_{n}, \bar{X}\left(t_{n}\right)\right), \partial_{\alpha} d \equiv \partial_{\alpha} d\left(t_{n}, \bar{X}\left(t_{n}\right)\right) .
$$

Here $\varphi \in \mathbb{R}^{d}$ is the solution of the discrete dual backward problem

$$
\begin{aligned}
& \varphi_{i}\left(t_{n}\right)=\partial_{i} c_{j}\left(t_{n}, \bar{X}\left(t_{n}\right)\right) \varphi_{j}\left(t_{n+1}\right), t_{n}<T, \\
& \varphi_{i}(T)=\partial_{i} g(\bar{X}(T)),
\end{aligned}
$$

with

$$
c_{i}\left(t_{n}, x\right) \equiv x_{i}+h_{n} a_{i}\left(t_{n}, x\right)+\Delta W_{n}^{\ell} b_{i}^{\ell}\left(t_{n}, x\right)
$$

and its first and second variation

$$
\begin{aligned}
\varphi_{i j}^{\prime} & \equiv \partial_{x_{j}\left(t_{n}\right)} \varphi_{i}\left(t_{n}\right) \equiv \frac{\partial \varphi_{i}\left(t_{n} ; \bar{X}\left(t_{n}\right)=x\right)}{\partial x_{j}} \\
\varphi_{i k m}^{\prime \prime}\left(t_{n}\right) & \equiv \partial_{x_{m}\left(t_{n}\right)} \varphi_{i k}^{\prime}\left(t_{n}\right) \equiv \frac{\partial \varphi_{i k}^{\prime}\left(t_{n} ; \bar{X}\left(t_{n}\right)=x\right)}{\partial x_{m}}
\end{aligned}
$$


which satisfy

$$
\begin{aligned}
\varphi_{i k}^{\prime}\left(t_{n}\right)= & \partial_{i} c_{j}\left(t_{n}, \bar{X}\left(t_{n}\right)\right) \partial_{k} c_{p}\left(t_{n}, \bar{X}\left(t_{n}\right)\right) \varphi_{j p}^{\prime}\left(t_{n+1}\right) \\
& +\partial_{i k} c_{j}\left(t_{n}, \bar{X}\left(t_{n}\right)\right) \varphi_{j}\left(t_{n+1}\right), t_{n}<T, \\
\varphi_{i k}^{\prime}(T)= & \partial_{i k} g(\bar{X}(T)),
\end{aligned}
$$

and

$$
\begin{aligned}
\varphi_{i k m}^{\prime \prime}\left(t_{n}\right)= & \partial_{i} c_{j}\left(t_{n}, \bar{X}\left(t_{n}\right)\right) \partial_{k} c_{p}\left(t_{n}, \bar{X}\left(t_{n}\right)\right) \partial_{m} c_{r}\left(t_{n}, \bar{X}\left(t_{n}\right)\right) \varphi_{j p r}^{\prime \prime}\left(t_{n+1}\right) \\
& +\partial_{i m} c_{j}\left(t_{n}, \bar{X}\left(t_{n}\right)\right) \partial_{k} c_{p}\left(t_{n}, \bar{X}\left(t_{n}\right)\right) \varphi_{j p}^{\prime}\left(t_{n+1}\right) \\
& +\partial_{i} c_{j}\left(t_{n}, \bar{X}\left(t_{n}\right)\right) \partial_{k m} c_{p}\left(t_{n}, \bar{X}\left(t_{n}\right)\right) \varphi_{j p}^{\prime}\left(t_{n+1}\right) \\
& +\partial_{i k} c_{j}\left(t_{n}, \bar{X}\left(t_{n}\right)\right) \partial_{m} c_{p}\left(t_{n}, \bar{X}\left(t_{n}\right)\right) \varphi_{j p}^{\prime}\left(t_{n+1}\right) \\
& +\partial_{i k m} c_{j}\left(t_{n}, \bar{X}\left(t_{n}\right)\right) \varphi_{j}\left(t_{n+1}\right), t_{n}<T \\
\varphi_{i k m}^{\prime \prime}(T)= & \partial_{i k m} g(\bar{X}(T)),
\end{aligned}
$$

respectively.

The previous result can also be directly applied to the particular case of deterministic time steps. Observe that the error expansion in Theorem 2.1 has the form

$$
E[g(X(T))-g(\bar{X}(T))]=E\left[\sum_{n=1}^{N} \bar{\rho}_{n} h_{n}^{2}\right]+\text { higher order terms }
$$

and due to the almost sure convergence of the density $\bar{\rho}_{n}$ as we refine the discretization, see [III], it is suitable for use in the adaptive algorithm.

The computational error in (10) naturally separates into the time discretization error and the statistical error

$$
\begin{aligned}
& E[g(X(T))]-\frac{1}{M} \sum_{j=1}^{M} g\left(\bar{X}\left(T ; \omega_{j}\right)\right) \\
= & (E[g(X(T))-g(\bar{X}(T))])+\left(E[g(\bar{X}(T))]-\frac{1}{M} \sum_{j=1}^{M} g\left(\bar{X}\left(T ; \omega_{j}\right)\right)\right) \\
\equiv & \mathcal{E}_{T}+\mathcal{E}_{S} .
\end{aligned}
$$

The time steps for the realizations of the approximate solution $\bar{X}$ are determined from statistical approximations of the time discretization error, $\mathcal{E}_{T}$, and the number, $M$, of realizations of $\bar{X}$ is determined from the statistical error, $\mathcal{E}_{S}$. The statistical error and the time discretization error are combined in order to bound the computational error $(20)$. Therefore we split a given error tolerance TOL into a statistical tolerance, $\mathrm{TOL}_{S}$, and a time discretization tolerance, $\mathrm{TOL}_{T}$. The computational work is roughly $\mathcal{O}(N \cdot M)=\mathcal{O}\left(\mathrm{TOL}_{T}^{-1} \mathrm{TOL}_{S}^{-2}\right)$, therefore we use

$$
\mathrm{TOL}_{T}=\frac{1}{3} \mathrm{TOL} \text { and } \mathrm{TOL}_{S}=\frac{2}{3} \mathrm{TOL},
$$

by minimizing $\mathrm{TOL}_{T}^{-1} \mathrm{TOL}_{S}^{-2}$ under the constraint $\mathrm{TOL}_{T}+\mathrm{TOL}_{S}=\mathrm{TOL}$.

From the central limit theorem, the statistical error is bounded by the following quantity, i.e. the event

$$
\left|\mathcal{E}_{S}(\bar{X} ; M)\right| \leq \mathrm{E}_{S}(\bar{X} ; M) \equiv c_{0} \frac{\mathcal{S}(\bar{X} ; M)}{\sqrt{M}}
$$


has probability close to one, where $\mathcal{S}(\bar{X} ; M)$ is the sample standard deviation of $\bar{X}$ and $c_{0}$ ia a constant related to the confidence interval.

The time discretization error is approximated by (19) and its contribution from each of the realizations controlled according to the adaptive algorithm described in Section [2.4.

2.3. An Error Expansion for PDEs. Consider a problem to compute a linear functional

$$
g(u):=\int_{D} u G d x
$$

for a given function $G \in L^{2}(D)$ and $u$ is the solution of a second order elliptic partial differential equation of the form

$$
-\operatorname{div}(a \nabla u)=f
$$

in a given open bounded domain $D \subset \mathbb{R}^{d}$ with Dirichlet boundary data $\left.u\right|_{\partial D}=0$.

The finite element approximation $u_{h}$, of $u$ in (23), is based on the standard variational formulation in the function set $V_{h}$ of continuous piecewise isoparametric bilinear quadrilateral functions in $H_{0}^{1}(D)$, using an adaptive quadrilateral mesh with hanging nodes cf. [I] . The Sobolev space $H_{0}^{1}(D)$ is the usual Hilbert space of functions on $D$, vanishing on $\partial D$, with bounded first derivatives in $L^{2}(D)$. Let $\mathcal{T}$ denote the set of convex quadrilaterals $K$ and let $h_{K}$ be the local mesh size, i.e. the length of the longest edge of $K$. Then the variational problems for $u \in H_{0}^{1}(D)$ and $u_{h} \in V_{h}$ are

$$
\begin{aligned}
\int_{D} a \nabla u \cdot \nabla v d x & =\int_{D} f v d x, \quad \forall v \in H_{0}^{1}(D), \\
\int_{D} a \nabla u_{h} \cdot \nabla v d x & =\int_{D} f v d x, \quad \forall v \in V_{h} .
\end{aligned}
$$

A central role in the dual weighted error representation for $g(u)-g\left(u_{h}\right)$ is played by the dual function $\varphi \in H_{0}^{1}(D)$ which satisfies

$$
\int_{D} a \nabla \varphi \cdot \nabla v d x=\int_{D} G v d x, \quad \forall v \in H_{0}^{1}(D) .
$$

Besides, its finite element approximation $\varphi_{h} \in V_{h}$, defined by

$$
\int_{D} a \nabla \varphi_{h} \cdot \nabla v d x=\int_{D} G v d x, \quad \forall v \in V_{h}
$$

is used to construct the error density $\bar{\rho}$ in Theorem 2.2 .

For general meshes the convergence of the error density does not hold, since the orientation of the elements varies. Thus, here the analysis considers the asymptotic behavior of the error density $\bar{\rho}$ for adaptive refinements, with general quadrilateral initial meshes: successive division of reference square elements into four similar squares generates hanging node meshes consisting of unions of structured adapted meshes, where each structured mesh has the domain of an initial element; viewed in the initial reference element the structured adaptive mesh is an adaptive hanging node mesh with square elements. We restrict the study to such unions of structured adaptive hanging node meshes. The use of quadrilaterals can directly be extended to higher space dimension using tensor reference elements. Other refinements using e.g. subdivision of a simplex, in three and higher dimensions cf. [4], generate new edges which are not parallel to the old and would require additional analysis. 
There is a smooth mapping of each initial element to a square, so that the refined initial element is mapped to a square hanging node mesh. Let $\mathcal{T}_{I}$ denote the subset of elements with an edge on the initial mesh. Theorem 2.2 states that the error density has a precise expansion using that the isoparametric bilinear coordinate transformation $X^{-1}:[0,1]^{2} \rightarrow K_{I}$ maps the square and the square hanging node mesh to the initial element $K_{I}$ and its refined hanging node quadrilateral mesh.

Let us now study the transformation of the variational formulation under such a mapping $X: K_{I} \rightarrow[0,1]^{2}$

$$
\sum_{i j} \int_{K_{I}}\left(a_{i j} \frac{\partial u_{h}}{\partial x_{j}} \frac{\partial v}{\partial x_{i}}-f v\right) d x=\int_{[0,1]^{2}}\left(a X^{\prime} u_{h}^{\prime} \cdot X^{\prime} v^{\prime}-f v\right) J d x^{\prime}
$$

where $X^{\prime}$ is the Jacobian of $X$ and $J$ is the Jacobian determinant. Here we abuse the notation by writing $v$ instead of $\left(v \circ X^{-1}\right)$ and similarly for $a, u_{h}$, and $f$, for $x \in K_{I}$. Besides, we write $v^{\prime}=\frac{\partial v}{\partial x_{i}^{\prime}}$ instead of $\frac{\partial\left(v \circ X^{-1}\right)}{\partial x_{i}^{\prime}}$.

Therefore the variational equation in the transformed coordinates, $x^{\prime}$, takes the same form with $a$ and $f$ replaced by $a^{*} \equiv J\left(X^{\prime}\right)^{t} a X^{\prime}$ and $f^{*} \equiv J f$, respectively. Note that $a^{*}$ and $f^{*}$ are as smooth on $X\left(K_{I}\right)$ as the functions $a$ and $f$ are on $K_{I}$. To avoid messy notation, we will not always use the prime notation for coordinates obviously in the reference elements; we will also avoid notation for the dependence of $X$ on the initial element $K_{I}$ and assume that we for a point $x \in D$ choose the mapping $X$ that corresponds to the initial element $K_{I}$ which contains $x$. We will use the set of transformed elements $\mathcal{T}^{\prime} \equiv\{X(K): K \in \mathcal{T}\}$.

To define the approximate error density, $\bar{\rho}$, we will use averages of second difference quotients as follows. Consider a function $w$ which is defined on a discretization of an interval $[0, L]$ with nodes $\left\{x_{j}: j=0, \ldots, \bar{N}+1\right\}=: \overline{\mathcal{N}}$, where $x_{0}=0$ and $x_{\bar{N}+1}=L$. Let $h_{+} \equiv x_{j+1}-x_{j}$ and $h_{-} \equiv x_{j}-x_{j-1}$ denote two consecutive edge sizes. Then define the average mesh size $\bar{h}$ and the difference quotients

$$
\begin{aligned}
\bar{h}_{j} & \equiv \frac{h_{+}+h_{-}}{2}=\frac{x_{j+1}-x_{j-1}}{2} \\
D w\left(x_{j}\right) & \equiv \frac{w\left(x_{j}+h_{+}\right)-w\left(x_{j}\right)}{h_{+}} \\
D^{2} w\left(x_{j}\right) & \equiv \frac{1}{\bar{h}_{j}}\left(\frac{w\left(x_{j}+h_{+}\right)-w\left(x_{j}\right)}{h_{+}}-\frac{w\left(x_{j}\right)-w\left(x_{j}-h_{-}\right)}{h_{-}}\right) .
\end{aligned}
$$

Define $\overline{D^{2} w} \in \mathbb{R}^{\bar{N}}$, implicitly as the solution $Y \in \mathbb{R}^{\bar{N}+2}$ of an auxiliary equation, i.e.

$$
\begin{aligned}
{\overline{D^{2} w_{n}}} & \equiv Y_{n}, \quad n=1, \ldots, \bar{N}, \text { where } \\
Y_{n}-\alpha^{2} D^{2} Y_{n} & =D^{2} w_{n}, \quad n=1, \ldots, \bar{N},
\end{aligned}
$$

with homogeneous Neumann boundary conditions, $Y_{0}=Y_{1}, Y_{\bar{N}}=Y_{\bar{N}+1}$. The work [6] reports numerical results of different alternative averages, including the fast nearest neighbor variant. The convergence proof requires $\alpha$ to be sufficiently large compared to the mesh size, cf. (34).

Let us define $\bar{h} D_{i}^{2} w$ as the difference quotients $\bar{h} D^{2} w$, in (27), with respect to the $x_{i}^{\prime}$ reference directions $i=1,2$, respectively, and analogously for $D_{i} w$. The 
approximate error density, $\bar{\rho}$, in the transformed coordinates is now defined by

$$
\bar{\rho}_{K} \equiv \frac{1}{48} \sum_{j=1}^{4}\left(a_{11}^{*} \overline{D_{1}^{2} u_{h}} \overline{D_{1}^{2} \varphi_{h}}+a_{22}^{*} \overline{D_{2}^{2} u_{h}} \overline{D_{2}^{2} \varphi_{h}}\right)\left(x_{j}^{K}\right)
$$

where $x_{1}^{K}, x_{2}^{K}, x_{3}^{K}, x_{4}^{K}$ are the four corners of the square $K \in \mathcal{T}^{\prime}$ illustrated in Figure [1.

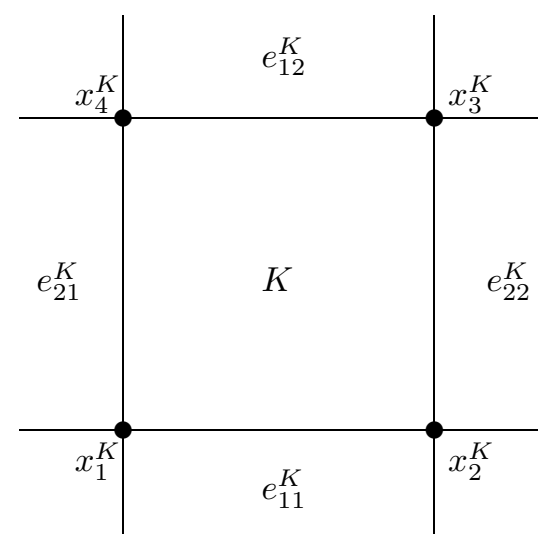

Figure 1 . Corners $x_{j}^{K}$ and edges $e_{i j}^{K}$ of a square $K \in \mathcal{T}^{\prime}$

Let $\mathcal{T}_{H}$ denote the subset of elements with hanging nodes in neighbors and let $\overline{\mathcal{T}}_{H} \equiv \bigcup_{K \in \mathcal{T}_{H}} K$. Let $W^{1, \infty}(D)$ denote the usual Sobolev space of functions with bounded first order derivatives in $L^{\infty}(D)$ and let $h_{\max }$ be the maximal edge length in the mesh of $V_{h}$. Sometimes we drop the set and write $W^{1, \infty}$ and $L^{\infty}$ also for functions in the reference set $[0,1]^{2}$. The main result in $[\overline{\boldsymbol{6}}]$ is

TheOREM 2.2. Assume that $a \in \mathcal{C}^{1}(\bar{D})$ and that the solutions $u \in \mathcal{C}^{3}(\bar{D}), \varphi \in$ $\mathcal{C}^{3}(\bar{D})$ of (23) and (25), respectively, are for some $\gamma \in(0,1)$ approximated uniformly with error

$$
\begin{aligned}
\left\|u-u_{h}\right\|_{W^{1, \infty}(D)}+\left\|\varphi-\varphi_{h}\right\|_{W^{1, \infty}(D)} & =\mathcal{O}\left(h_{\max }\right), \\
\left\|u-u_{h}\right\|_{L^{\infty}(D)}+\left\|\varphi-\varphi_{h}\right\|_{L^{\infty}(D)} & =\mathcal{O}\left(h_{\max }^{2 \gamma}\right),
\end{aligned}
$$

using piecewise isoparametric bilinear quadrilateral elements and a refined mesh, with at most one hanging node per edge, obtained by successively dividing the reference square elements into four similar squares. Assume also that all second order difference quotients of $u_{h}$ are uniformly bounded and that the total area of the elements with a hanging node on a neighbor or with an edge on the initial mesh is asymptotically zero:

$$
\int_{\overline{\mathcal{T}}_{H} \cup \overline{\mathcal{T}}_{I}} d x=o(1), \quad \text { as } h_{\max } \rightarrow 0+.
$$

Then the global error has the expansion

$$
g(u)-g\left(u_{h}\right)=\sum_{K \in \mathcal{T}^{\prime}}\left(\bar{\rho}_{K}+\mathcal{O}\left(h_{\max }^{\gamma} / \alpha+\alpha\right)\right) h_{K}^{4}+\mathcal{O}\left(h_{\max }\right) \int_{\overline{\mathcal{T}}_{H} \cup \overline{\mathcal{T}}_{I}} h_{K} d x
$$


with uniformly convergent computable leading order error density $\bar{\rho}$, defined by (29) and (27) $-(28)$ for $\alpha^{-1}=o\left(h_{\max }^{-\gamma}\right)$, satisfying

$$
\bar{\rho}=\widetilde{\rho}+\mathcal{O}\left(h_{\max }^{\gamma} / \alpha+\alpha\right)
$$

where

$$
\widetilde{\rho} \equiv \frac{1}{12}\left(a_{11}^{*} \frac{\partial^{2} u}{\partial x_{1}^{2}} \frac{\partial^{2} \varphi}{\partial x_{1}^{2}}+a_{22}^{*} \frac{\partial^{2} u}{\partial x_{2}^{2}} \frac{\partial^{2} \varphi}{\partial x_{2}^{2}}\right)
$$

is evaluated in the transformed coordinates on $[0,1]^{2}$.

Note that the convergence of $\bar{\rho}$ is uniform while the convergence of $\check{\rho}$, defined by $g(u)-g\left(u_{h}\right) \equiv \sum_{K \in \mathcal{T}}, \check{\rho} h_{K}^{4}$, is in $L^{1}(D)$ by assumption (31). It is important to notice that our restriction of the data, required by $u, \varphi \in \mathcal{C}^{3}(\bar{D})$, includes examples with substantial adaptive gain. Section 3 shows that the optimal number of adaptive elements is $N^{o p t} \simeq \mathrm{TOL}^{-1}\|\bar{\rho}\|_{L^{\frac{d}{d+2}}}^{d / 2}$, while the number of uniform elements becomes $N^{u n i} \simeq \mathrm{TOL}^{-1}\|\bar{\rho}\|_{L^{1}}^{d / 2}$ to achieve the same error TOL. Although $u, \varphi \in \mathcal{C}^{3}(\bar{D})$ their norms in these spaces may be large so that $\|\bar{\rho}\|_{L^{\frac{d}{d+2}}} \ll\|\bar{\rho}\|_{L^{1}}$.

In general, second order difference quotients of the interpolant on meshes with hanging nodes do not converge uniformly on $D$ and this why the averages are needed $[\mathbf{6}]$.

Finite element approximations of the coercive linear problems (23) and (25), with piecewise isoparametric bilinear quadrilateral elements, satisfy the estimate

$$
\begin{aligned}
\left\|u-u_{h}\right\|_{L^{\infty}}+\left\|\varphi-\varphi_{h}\right\|_{L^{\infty}} & =\mathcal{O}\left(h_{\text {max }}^{2} \log h_{\text {max }}^{-1}\right), \\
\left\|u-u_{h}\right\|_{W^{1, \infty}} & =\mathcal{O}\left(h_{\max }\right),
\end{aligned}
$$

provided $u, \varphi \in \mathcal{C}^{2}(\bar{D})$, see [2], [3]. This estimate and the proof of Theorem 2.2 imply that the choice

$$
\alpha^{-1}=o\left(\left(h_{\max } \sqrt{\log h_{\max }^{-1}}\right)^{-1}\right),
$$

yields convergent error densities.

Theorem 2.2 proves that the error expansion

$$
g(u)-g\left(u_{h}\right)=\sum_{K \in \mathcal{T}^{\prime}}\left(\bar{\rho}_{K}+\mathcal{O}\left(\frac{h_{\max }^{\gamma}}{\alpha}+\alpha\right)\right) h_{K}^{2+d}+\mathcal{O}\left(h_{\max }\right) \sum_{K \in \mathcal{T}_{H}^{\prime} \cup \mathcal{T}_{I}^{\prime}} h_{K}^{1+d}
$$

has a well defined leading order error density $\bar{\rho}$ which converges uniformly as $h_{\max } \rightarrow 0+$. We now assume that $\alpha$ has been chosen such that

$$
\frac{h_{\max }^{\gamma}}{\alpha}+\alpha=\mathcal{O}\left(h_{\max }^{\widehat{\gamma}}\right),
$$

where $\widehat{\gamma}>0$.

2.4. The Adaptive Algorithm. To motivate the approximate equidistribution of the error indicators in an adaptive algorihtm, consider an asymptotic error expansion

$$
\text { error } \simeq \sum_{n} \rho_{n} h_{n}^{p+d},
$$

where $h$ is the local isotropic mesh size and $\rho$ is independent of $h$. The number of elements that corresponds to a mesh with size $h$ can be determined by

$$
N(h) \equiv \int_{D} \frac{d x}{h^{d}(x)} .
$$


It seems hard to use the sign of the error indicators for constructing the mesh. Instead, we minimize the number of elements $N$ in (37) under the more stringent constraint

$$
\sum_{n=1}^{\bar{N}}\left|\rho_{n}\right| h_{n}^{d+p}=\int_{D}|\rho(x)| h^{p} d x=\text { TOL }
$$

with $D=[0, T]$ and $d=1$ for ODEs and SDEs. The global order of convergence satisfies $p=1$ for the Euler-Maruyama SDE approximation and $p=2$ for the $d$-linear finite elements approximations used here. A standard application of a Lagrange multiplier yields the optimum

$$
|\rho|\left(h^{*}\right)^{d+p}=\text { constant }
$$

and

$$
h^{*} \equiv \frac{\mathrm{TOL}^{\frac{1}{p}}}{|\rho|^{\frac{1}{d+p}}}\left(\int_{D}|\rho(x)|^{\frac{d}{d+p}} d x\right)^{-\frac{1}{p}} .
$$

This condition is optimal only for density functions $\rho$ with one sign, and in the PDE case, for meshes with shape regular elements, i.e. non stretched elements. To use the sign of the density or orientation of stretched elements in an optimal way is not considered here.

In the adaptive algorithm below we will use the positive approximate error density $\hat{\rho}_{K}$ defined by

$$
\left.\hat{\rho}\right|_{K} \equiv \hat{\rho}_{K} \equiv \min \left(\max \left(\left|\bar{\rho}_{K}\right|, \delta\right), \mathrm{TOL}^{-r}\right)
$$

with $r>0$ and where the lower bound, $\delta>0$, is chosen according to

REMARK 2.3 (Lower bound for the error density).

$$
\delta \equiv \mathrm{TOL}^{\bar{\gamma}}
$$

where the parameter $\bar{\gamma}$ is $0<\bar{\gamma}<1 /(p+1)$ for ODEs, $\bar{\gamma}=1 / 9$ for SDEs, and for PDEs it is chosen such that satisfies the two lower bounds

$$
\bar{\gamma}<\frac{\widehat{\gamma}}{\widehat{\gamma}+2} \text { and } \int_{\overline{\mathcal{T}}_{H} \cup \overline{\mathcal{T}}_{I}} d x / \delta=o(1) \text { as TOL } \rightarrow 0+,
$$

and the upper bound $\delta=o(1)$ as TOL $\rightarrow 0+$. The lower bounds on $\delta>0$ are motivated by the requirements that $h_{\max } \rightarrow 0$ as $\mathrm{TOL} \rightarrow 0$, that the bounds for the error density in (50) hold and that the error from hanging node elements becomes asymptotically negligible, see Theorem 3.2. The convergence of $\hat{\rho}$ towards the exact density requires the upper bound $\delta \rightarrow 0$.

The goal of the adaptive algorithm described below is to construct a mesh such that

$$
\hat{\rho}_{n} h_{n}^{d+p} \approx \frac{\mathrm{TOL}}{N}, n=1, \ldots, N,
$$

which is an approximation of the optimal (39). Let the index $[k]$ refer to the refinement level in the sequence of adaptively refined meshes. For a mesh with elements $\left\{K_{1}, K_{2}, K_{3}, \ldots, K_{N}\right\}$, we consider the piecewise constant error density and mesh functions $\left.\rho\right|_{K_{n}} \equiv \rho_{n} \equiv \rho_{K_{n}},\left.\quad \hat{\rho}\right|_{K_{n}} \equiv \hat{\rho}_{n} \equiv \hat{\rho}_{K_{n}}$ and $\left.h\right|_{K_{n}} \equiv h_{n} \equiv h_{K_{n}}$. To achieve (44) let $s_{1} \approx 1$ be a given constant, start with an initial mesh of size 
$h[1]$ and then specify iteratively a new mesh $h[k+1]$, from $h[k]$, using the following dividing strategy:

(45)

$$
\begin{aligned}
& \text { for all intervals (elements) } n=1,2, \ldots, N[k] \\
& \bar{r}_{n}[k] \equiv \hat{\rho}_{n}[k]\left(h_{n}[k]\right)^{d+p} \\
& \text { if } \quad \bar{r}_{n}[k]>s_{1} \frac{\mathrm{TOL}}{N[k]} \text { then } \\
& \text { mark interval (element) } n \text { for division. } \\
& \text { (In addition, for the PDE case mark recursively all neighbors } \\
& \text { that need division due to the hanging node constraint: } \\
& \quad \text { at most one hanging node per edge.) } \\
& \text { endif } \\
& \text { endfor }
\end{aligned}
$$

divide every marked interval (element) into $2^{d}$ uniform sub intervals (elements).

With this dividing strategy, it is natural to use the stopping criterion:

$$
\text { if }\left(\max _{1 \leq n \leq N[k]} \bar{r}_{n}[k] \leq S_{1} \frac{\text { TOL }}{N[k]}\right) \text { then stop. }
$$

Here $S_{1}$ is a given constant, with $S_{1}>s_{1} \approx 1$, determined more precisely as follows: we want that the maximal error indicator decays quickly to the stopping level $S_{1} \mathrm{TOL} / N$, but when almost all error indicators $\bar{r}_{n}$ satisfy $\bar{r}_{n}<s_{1} \frac{\mathrm{TOL}}{N}$ the reduction of the error may be slow. Theorem 3.1] shows that a slow reduction is avoided if $S_{1}$ satisfies (51).

Remark 2.4 (SDE case: Stochastic Time Steps). Let $\bar{g} \equiv \sum_{j=1}^{M} g\left(\bar{X}(T) ; \omega_{j}\right)$ be the sample average approximation of the expected value $E[g(X(T))]$ and let $\bar{N}[m]$ be the sample average of the final number of time steps in the $m$-th batch of $M[m]$ realizations. In this case (45), is used iteratively for each of the realizations, $j=1, \ldots, M[m]$, with $\mathrm{TOL}_{T}$ instead of TOL and with $\bar{N}$ instead of $N$, see [10]. Replacing the integrals $\int_{D} \ldots d x$ by $\iint \ldots d t d P=E \int \ldots d t$ formally motivates the equidistribution of the error indicators for each realization of the Brownian motion.

\section{Convergence Rates for the Adaptive Mesh Algorithm}

This section presents results on the stopping, accuracy and efficiency properties of adaptive algorithm introduced in Section 2 .

3.1. Adaptive Refinements and Stopping. To analyze the decay of the maximal error indicator, it is useful to understand the variation of the density $\hat{\rho}$ at different refinement levels, in particular we will consider an element or time step $K[k]$ and its parent on a previous refinement level, $p(K, k)$, with the corresponding error density $\hat{\rho}(K)[p(K, k)]$. It is possible to verify that the choice (42) of $\delta$ implies that $h_{\max } \rightarrow 0$ as TOL $\rightarrow 0+$, see [6],$[\boldsymbol{8}]$, [10]. Hence Theorem 2.2 shows, for the $\mathrm{PDE}$, that there is a limit error density $\widetilde{\rho}$ such that

$$
\check{\rho} \stackrel{L^{1}}{\longrightarrow} \widetilde{\rho}, \bar{\rho} \rightarrow \widetilde{\rho} \text { and } \hat{\rho} \rightarrow|\widetilde{\rho}|, \text { as TOL } \rightarrow 0+.
$$


Similarly, the choice (42) of $\delta$ is used to show in [8] for ODEs that

$$
\hat{\rho} \rightarrow|\widetilde{\rho}|, \text { as TOL } \rightarrow 0+,
$$

and in [10] that for each realization of the SDE, with $0<\alpha<1 / 2$,

$$
\lim _{\text {TOL } \rightarrow 0+} h_{\max }^{-\alpha}(\hat{\rho}-|\widetilde{\rho}|)=0, \quad \text { almost surely. }
$$

A consequence of the uniform convergence $\hat{\rho} \rightarrow|\widetilde{\rho}|$, as TOL $\rightarrow 0+$, and (41) is that for all elements $K$ and all refinement levels $k$ there exists positive functions $\hat{c}$ and $\hat{C}$ close to 1 for sufficiently refined meshes, such that the error density satisfies

$$
\begin{gathered}
\hat{c}(K) \leq \frac{\hat{\rho}(K)[p(K, k)]}{\hat{\rho}(K)[k]} \leq \hat{C}(K), \\
\hat{c}(K) \leq \frac{\hat{\rho}(K)[k-1]}{\hat{\rho}(K)[k]} \leq \hat{C}(K),
\end{gathered}
$$

provided $\max _{K, k} h_{K}[k]$ is sufficiently small. In other words, (50) holds with e.g. $\hat{c}=2^{-1}=\hat{C}^{-1}$ for sufficiently small $\max _{K, k} h_{K}[k]$. For SDEs the functions $\hat{c}$ and $\hat{C}$ are close to 1 , almost surely.

THEOREM 3.1 (Stopping). Suppose the adaptive algorithm uses the strategy (45) -(46). Assume that $\hat{c}$ satisfies (50), for the elements or time steps corresponding to the maximal error indicator on each refinement level, and that

$$
S_{1} \geq \frac{2^{d}}{\hat{c}} s_{1}, \quad 1>\frac{\hat{c}^{-1}}{2^{d+p}} .
$$

Then each refinement level either decreases the maximal error indicator with the factor

$$
\max _{1 \leq n \leq N[k+1]} \bar{r}_{n}[k+1] \leq \frac{\hat{c}^{-1}}{2^{d+p}} \max _{1 \leq n \leq N[k]} \bar{r}_{n}[k],
$$

or stops the algorithm.

Here, the global order of convergence is $p=1$ for the Euler-Maruyama SDE approximation and $p=2$ for the $d$-linear finite elements approximations.

3.2. Accuracy of the Adaptive Algorithm. The adaptive algorithm guarantees that the estimate of the global error is bounded by a given error tolerance, TOL. An important question is whether the true global error is bounded by TOL asymptotically. Using the upper bound (46) of the error indicators and the convergence of $\rho$ and $\bar{\rho}$ in Theorem 2.2, or the convergence (48), (49) respectively, the global error has the following estimate.

Theorem 3.2 (Accuracy). Suppose (41)-(42) hold and that, for PDEs (36) and the assumptions of Theorem 2.9 hold, or, for ODEs and SDEs, (48) and (49) holds, respectively. Then the adaptive algorithm (45)-(46) satisfies

$$
\begin{array}{r}
\limsup _{\mathrm{TOL} \rightarrow 0+}\left(\mathrm{TOL}^{-1}|g(X(T))-g(\bar{X}(T))|\right) \leq S_{1}, \quad \text { for the } O D E, \\
\limsup _{\mathrm{TOL} \rightarrow 0+}\left(\mathrm{TOL}^{-1}\left|g(u)-g\left(u_{h}\right)\right|\right) \leq S_{1}, \quad \text { for the PDE, }
\end{array}
$$


and, for the SDE, with the number of realizations $M$ and any $c_{0}>0$ determined by (22),

$$
\liminf _{\mathrm{TOL} \rightarrow 0+} P\left(\frac{1}{\mathrm{TOL}}\left|E[g(X(T))]-\frac{1}{M} \sum_{j=1}^{M} g\left(\bar{X}\left(T ; \omega_{j}\right)\right)\right| \leq \frac{S_{1}+2}{3}\right) \geq \int_{-c_{0}}^{c_{0}} \frac{e^{-x^{2} / 2}}{\sqrt{2 \pi}} d x .
$$

3.3. Efficiency of the Adaptive Algorithm. An important issue for the adaptive method is its efficiency; we want to determine a mesh with as few elements or time steps as possible providing the desired accuracy. From the definition (37) and the optimality condition (40), the number of optimal adaptive elements, $N^{\text {opt }}$, satisfies

$$
N^{\mathrm{opt}}=\int_{D} \frac{d x}{\left(h^{*}(x)\right)^{d}}=\frac{1}{\operatorname{TOL}^{\frac{d}{p}}}\left(\int_{D}|\rho[k](x)|^{\frac{d}{d+p}} d x\right)^{\frac{d+p}{p}}=\frac{1}{\operatorname{TOL}^{\frac{d}{p}}}\|\rho\|_{L^{\frac{d}{d+p}}}^{\frac{d}{p}} .
$$

On the other hand, for the uniform mesh with elements $h=$ constant, the number of elements, $N^{\text {uni }}$, to achieve $\sum_{i=1}^{N}\left|\rho_{i}\right| h^{d+p}=$ TOL becomes

$$
N^{\mathrm{uni}}=\int_{D} \frac{d x}{h^{d}(x)}=\frac{\int_{D} d x}{\mathrm{TOL}^{\frac{d}{p}}}\left(\int_{D}|\rho[k](x)| d x\right)^{\frac{d}{p}}=\frac{\int_{D} d x}{\mathrm{TOL}^{\frac{d}{p}}}\|\rho\|_{L^{1}}^{\frac{d}{p}} .
$$

Hence, the number of uniform elements is measured in the $L^{1}$-norm while the optimal number of elements is measured in the $L^{\frac{d}{d+p}}$ quasi-norm. Jensen's inequality implies $\|f\|_{L^{\frac{d}{d+p}}} \leq\left(\int_{D} d x\right)^{\frac{p}{d}}\|f\|_{L^{1}}$, therefore an adaptive method may use fewer elements than the uniform element size method. For the SDE we get the optimal expected number of adaptive steps $E\left[N^{\mathrm{opt}}\right]=\frac{1}{\text { TOL }}\left(E \int_{0}^{T} \sqrt{|\rho|} d t\right)^{2}=$ $\frac{1}{\text { TOL }}\|\rho\|_{L^{\frac{1}{2}(d t d P)}}$ while with uniform time steps $E\left[N^{\text {uni }}\right]=\frac{T}{\text { TOL }} \int_{0}^{T} E|\rho| d t$.

The following theorem uses a lower bound of the error indicators, obtained from the refinement criterion (45) for the refined parent error indicator and the ratio of the error density (50), to show that the algorithm (45)-(46) generates a mesh which is optimal, up to a multiplicative constant independent of the data. In order to guarantee that, for sufficiently small TOL, all elements on the initial mesh are refined, the initial mesh size is assumed to obey

$$
h_{K}[1] \geq \mathrm{TOL}^{s}
$$

where the parameter $s$ has the upper bound $s<\frac{1-\bar{\gamma}}{p}$ and the lower bounds $0<s$, for ODEs and SDEs, and $\frac{\bar{\gamma}}{\hat{\gamma}}<s$, for PDEs.

Theorem 3.3 (Efficiency). Assume that $\hat{C}=\hat{C}(t), \hat{C}(t, \omega)$ or $\hat{C}(x)$ satisfies (50) for all elements at the final refinement level, that the assumptions of Theorem 3.9 hold, and that the initial mesh satisfies (55) for all elements $K$. Then there exists a constant $C>0$, bounded by $\left(\frac{2^{d+p}}{s_{1}}\right)^{\frac{d}{p}}$, such that, for sufficiently small TOL, the final number of adaptive time steps or elements $N$, of the algorithm (45)-(46) for ODEs or PDEs, satisfies

$$
\left(\operatorname{TOL}^{\frac{d}{p}} N\right) \leq C\|\hat{C} \hat{\rho}\|_{L^{\frac{d}{d+p}}}^{\frac{d}{p}} \leq C\left(\max _{x \in D} \hat{C}(x)^{\frac{d}{p}}\right)\|\hat{\rho}\|_{L^{\frac{d}{d+p}}}^{\frac{d}{p}},
$$


and

$$
\begin{aligned}
& \lim _{\mathrm{TOL} \rightarrow 0+}\|\hat{\rho}\|_{L^{\frac{d}{d+p}}}=\|\widetilde{\rho}\|_{L^{\frac{d}{d+p}}}, \\
& \lim _{\mathrm{TOL} \rightarrow 0+} \max _{x \in D} \hat{C}(x)^{\frac{d}{p}}=1,
\end{aligned}
$$

i.e. the number of elements is asymptotically optimal up to the problem independent factor $C \leq\left(\frac{2^{d+p}}{s_{1}}\right)^{\frac{d}{p}}$. For the SDE case the final sample average $\bar{N}[m]=$ $\frac{1}{M[m]} \sum_{j=1}^{M[m]} N\left(\omega_{j}\right)$ of the number of adaptive steps of the algorithm (45) -(46) satisfies

$$
\frac{\mathrm{TOL}_{T} \bar{N}[m]^{2}}{\bar{N}[m-1]}<C^{2}\left(\int_{0}^{T} \frac{1}{M[m]} \sum_{j=1}^{M[m]} \sqrt{\hat{\rho} \hat{C}} d t\right)^{2}
$$

and asymptotically

$$
\limsup _{\operatorname{TOL}_{T} \rightarrow 0+} \operatorname{TOL}_{T} E[N] \leq C^{2}\|\widetilde{\rho}\|_{L^{\frac{1}{2}}(d t d P)}
$$

\section{References}

[1] R. Becker and R. Rannacher, An optimal control approach to a posteriori error estimation in finite element methods, Acta Numerica, (2001), 1-102.

[2] S. C. Brenner and L. R. Scott, The mathematical theory of finite element methods, Texts in Applied Mathematics 15, Springer-Verlag, New York, 1994.

[3] F. Christian and G. Santos, A posteriori estimators for nonlinear elliptic partial differential equations, J. Comput. Appl. Math., 103 (1999), 99-114.

[4] Edelsbrunner, H.; Grayson, D. R., Edgewise subdivision of a simplex. ACM Symposium on Computational Geometry (Miami, FL, 1999). Discrete Comput. Geom., 24 (2000), 707-719.

[5] K.-S. Moon, Convergence rates of adaptive algorithms for deterministic and stochastic differential equations, (Licentiate thesis, ISBN 91-7283-196-0, Royal Institute of Technology, 2001) http://www.nada.kth.se/ moon

[6] K.-S. Moon, A. Szepessy, E. von Schwerin and R. Tempone, Convergence Rates for an Adaptive Dual Weighted Residual Finite Element Algorithm, www.nada.kth.se/ szepessy.

[7] K.-S. Moon, A. Szepessy, E. von Schwerin and R. Tempone, Adaptive Monte Carlo algorithm for stopped diffusion.

[8] K.-S. Moon, A. Szepessy, R. Tempone and G.E. Zouraris, Convergence rates for adaptive approximation of ordinary differential equations, Numer. Math. 96 (2003), 99-129.

[9] K.-S. Moon, A. Szepessy, R. Tempone and G.E. Zouraris, A variational principle for adaptive approximation of ordinary differential equations Numer. Math. 96 (2003), 131-152.

[10] K.-S. Moon, A. Szepessy, R. Tempone and G.E. Zouraris, Convergence rates for adaptive weak approximation of stochastic differential equations, Preprint 2002.

[11] K.-S. Moon, A. Szepessy, R. Tempone and G.E. Zouraris, Hyperbolic differential equations and adaptive numerics, in Theory and numerics of differential equations (Eds. J.F. Blowey, J.P. Coleman and A.W. Craig, Durham 2000, Springer Verlag, 2001)

[12] A. Szepessy, R. Tempone and G. E. Zouraris, Adaptive weak approximation of stochastic differential equations, Comm. Pure Appl. Math., 54 (2001), 1169-1214.

[13] D. Talay and L. Tubaro, Expansion of the global error for numerical schemes solving stochastic differential equations, Stochastic Anal. Appl., 8, 483-509, 1990. 
Department of Mathematics, University of Maryland, College Park MD 207424015, USA

E-mail address: moon@math.umd.edu

Institutionen för Numerisk Analys och Datalogi, Kungl. Tekniska Högskolan, S10044 Stockholm, Sweden.

E-mail address: schwerin@nada.kth.se

Matematiska Institutionen, Kungl. Tekniska Högskolan, S-100 44 Stockholm, SweDEN.

E-mail address: szepessy@nada.kth.se

Institute for Computational and Engineering Sciences (ICES), The University of Texas at Austin, 1 University Station C0200, Austin, Texas 78712, USA.

E-mail address: rtempone@ices.utexas.edu 